Published in Ehrensberger-Dow M. \& Englund Dimitrova B. (eds) Exploring the Situational Interface of Translation and Cognition. Benjamins Current Topics 101. Amsterdam: John Benjamins. ISBN: 978-90-272-6336-0 .

https://dx.doi.org/10.1075/bct.101.05hok

\title{
Affect as a Hinge: The Translator's Experiencing Self as a Sociocognitive Interface
}

Sari Hokkanen and Kaisa Koskinen

Sari Hokkanen

University of Tampere

School of Language, Translation and Literary Studies

FI-33014 UNIVERSITY OF TAMPERE

Finland

sari.hokkanen@uta.fi

Kaisa Koskinen

University of Eastern Finland

Foreign Languages and Translation Studies

PO Box 111

80101 JOENSUU

Finland

kaisa.koskinen@uef.fi 


\begin{abstract}
Affect, understood here as embodied meaning-making, offers one useful point of departure in studying translation as an activity that involves both cognitive and social processes, because it functions as a hinge between subjective understandings and social environments. We approach affects related to translating with the theoretical framework of the translator's experiencing self, defined here as the perception that translators have of themselves, based on lived and embodied experience. In other words, we suggest that the study of affect in relation to translating should pay attention to translators' own processes of meaningmaking. Since the processes of human meaning-making, including self-construction, have been argued to rest upon narrative practice, we furthermore argue for a narrative approach to studying affects. We illustrate the methodological opportunities provided by a narrative approach to affect with three cases deriving from three different research projects.
\end{abstract}

Keywords: affect, experiencing self, narratives, methodology, laughter, autoethnography 


\section{Introduction}

Recently, researchers interested in translators' cognitive processes and those studying translation as a socio-historical, contextualized activity have increasingly found themselves on shared ground. ${ }^{1}$ With increasing attention to the situated and relational nature of cognition on the one hand, and to the experiencing self as a key agent in any context of human activity on the other hand (most visible in the continued popularity of the habitus concept), process researchers and sociologists of translation have begun to recognize their shared interests (Hubscher-Davidson 2011). However, cooperation between these two strands of Translation Studies will also need to address significant differences in research traditions, ontological presuppositions, and accepted methodologies.

In this article, we address the issue of methodology. We propose a particular avenue of research, one that focuses on affect. To illustrate how the notion of affect can be employed as a bridging research tool, we report three cases and discuss three methods that we have used ourselves. Intentionally, the methods are different from one another in terms of theoretical and ontological commitments, researcher engagement, social vs. personal focus, and limited vs. holistic analysis. The three cases are: analysis of laughter as a tool for negotiating professional identity in a translation department; translators’ emotional narratives as indicators of their relations to technology; and autoethnography as a methodological framework within which the researcher's own emotional and embodied experiences are analysed to understand interpreting in church.

Our basic argument is that interrogating the experiencing self of the translator offers one useful point of departure in studying translation as an activity that involves both cognitive and social processes in complex interaction. As emotions and affects are essential elements of human meaning-making, we further argue that focusing on affect offers methodological avenues for understanding and explaining these experiences. We approach this proposition from both a theoretical and a methodological point of view. We begin with the basic tenet of Situated Cognition, introduced to Translation Studies by Risku (e.g. 2002), that translation is not only carried out by an individual translator's brain, but by

\footnotetext{
${ }^{1}$ Throughout the paper, we use the terms translation and translator to refer to both written translation and interpreting for reasons of readability.
} 
systems of "people, their specific social and physical environments and all their cultural artefacts” (Risku 2002, 530). As Risku notes, the move of focus from the brain into such systems also highlights the role of embodiment: "our body and its perceptive organs not only absorb information, they also filter and structure it” $(2002,528)$. Affects function as one such structuring element: our gut reactions direct our interpretations of the situational factors as endearing, disgusting, dangerous, or safe. By modulating our signals of affect (Koskinen 2012), we connect to others operating in that same context, and our interpretations of others' affects guide our coexistence with them.

Affect is a complex psychobiological and sociocognitive phenomenon. In social psychology, the current psychological understanding of affect has been summarized as follows:

The picture that psychology and neuroscience typically now paints of affect is of a highly dynamic, interacting composite or assemblage of autonomic bodily responses (e.g. sweating, trembling, blushing), other bodily actions (approaching or avoiding), subjective feelings and other qualia, cognitive processing (e.g. perception, attention, memory, decision-making), the firing and projecting of neural circuits (e.g. from the thalamus to the cortex and the amygdala), verbal reports (from exclamations to narratives) and communicative signals such as facial expressions.

(Wetherell 2012, 62; emphasis in the original)

Affect, in other words, is simultaneously private and internal, and collective and social. This dual nature allows us to position it methodologically as a hinge between cognitive and social approaches to translation. The multifaceted nature of affect allows for numerous theoretical and methodological approaches. Our use of affect is restricted by the choice to focus on the individual's experiencing self through which affect is examined. Here, the self refers to the individual as perceived by him/herself (Mead 1937/1967). Thus, we do not discuss affect as a primarily physiological phenomenon, which could be detected and analysed without engaging with the individual's perspective, but as something that the individual can be conscious of and also express to others. While the emphasis of affect has led some social scientists to celebrate the possibilities of eschewing participants' accounts in favour of assumedly more reliable physiological data (see Wetherell 2012, 19-21), we do not believe that the complexities of affective relations can be understood without a fine- 
grained analysis of the discourses the participants use to make sense of their situation, or that lived experiences can be reduced to measurements of bodily functions only. As a result of this methodological choice, our three cases are all based on narrated affect in one way or another. In this paper, we treat narratives as a component of human meaning-making, in which individual experiences are explained by drawing from socially-available storylines (Holstein and Gubrium 2000). Therefore, our interest lies less in the way participants’ narratives relate to actual events and emotions, and more in the way narratives are socially situated and constructed.

The article begins with a review of previous studies linked to affect in Translation Studies (section 2). We then move on to discuss the nature of affect and the experiencing self as understood in this article as well as to argue for narrative approaches as a way of teasing out affects and experiences in their social and cognitive complexity (section 3). In section 4, we briefly describe three research projects we have conducted. These are not intended to stand as exemplary cases or models to be followed as such. Rather, we hope that they serve as sources of inspiration regarding the rich array of opportunities for future study. We discuss this methodological potential in the concluding section.

\section{Review of Previous Studies on Affect in Translation Studies}

The role of affect in the process of translation has been previously acknowledged by only a few Translation Studies scholars (for an overview, see Hubscher-Davidson 2013a). ${ }^{2}$ Among the early writers of translation from an affective perspective is Robinson, who argued for a "somatic” view on translation. Somatics, Robinson (1991, x) argues, is explicitly physical; it means "the visceral processes and the limbic system, particularly the 'emotional'”. He describes "the ways in which our body 'signals' to us what we know and how we should act on it," so that we "know in our gut" what we have to do and say. This physiological view of affect is causal: an outside stimulus makes our body react in a particular way. Methodologically, this physiological layer can be studied empirically with

\footnotetext{
${ }^{2}$ In this review we exclude studies examining affect as an object of translation or as a textual element, i.e., studies that take affect as a property of the text that is translated, and not as a feature of translators themselves or the receivers of translations. While these studies can be seen as related, their objectives and methodologies are quite different from our present interests.
} 
tools such as the EEG, tomography and pupillometrics (pupil size is known to respond to positive and negative affects), bringing questions of affect in contact with recent process studies (see O’Brien 2011, 5-6). Affectivity can also be operationalized in terms of personality traits, such as emotional intelligence (Hubscher-Davidson 2013b). As argued by Hubscher-Davidson (2013b, 333), several aspects of professional translation such as the ability to recognize clients' needs and transfer others' perspectives into new contexts are related to emotional intelligence. Thus, research on emotional intelligence can offer valuable new insights, especially in studies contrasting different groups of translators and explaining successful practice.

However, affect is not only physiological or a personal trait, but also a contextual and interactive phenomenon. Our affects are also shaped by the affects of others in the same context, and our past and present social experiences, making causalities complex and difficult to determine. Another option might thus entail a return to verbalizations like thinkaloud protocols (TAPs), to look for clues of the affects underlying translators' decisionmaking (this is the approach of Tirkkonen-Condit and Laukkanen 1996). Yet another approach, employed by Lehr (2014), is to combine an inquiry into translators' views with a textual study of finished translations in order to trace the effects of translators' affective engagement with the source text on the translated product. Lehr suggests that personal engagement and heightened affect, whether positive or negative, towards the source text may increase overall translation quality.

As reception studies have become popular, especially in the fields of audio-visual and literary translation, the affective elements of viewing or reading have also gained some recognition. Issues such as the reception of humour in film translation have been studied, but the often-employed quantitative questionnaire method creates an element of distance between viewers' affects and the observer, even in studies which aim at capturing signals of affect such as smiling or laughter (see e.g. Rossini and Chiaro 2010). Reception studies, logically, focus on the audience, not on the translators. In a move relevant for our present purposes, Pérez-González (2012) brings to the forefront the group affinity between viewers and (amateur) subtitlers. He emphasizes the role of affectivity and performativity in subtitles as they intervene with both the articulation and the reception - the 'spectatorial experiences' - of the audio-visual semiotic ensemble. The relevance of the affective 
involvement of both subtitlers and viewers is observed, but the article does not include any attempt at providing empirical evidence of their experiences.

The field of interpreting research has also identified affect as one factor of interpreters’ work. For example Baraldi and Gavioli $(2007,171)$ argue that in medical encounters the interpreter acts as a “responder” to the primary speakers' affective states, instead of merely transmitting verbal messages. Nevertheless, as suggested by Cirillo (2010, 72), the emotional cues expressed by all parties in an interpreted medical encounter are heavily influenced by the social context at hand. Furthermore, as discussed by Bontempo and Malcolm (2012, 112), interpreters in healthcare environments are “empathically affected” when they relay others’ emotional and possibly traumatic experiences in the first person. This, the authors argue, is one reason for interpreters' elevated risk of vicarious trauma, making affect an important issue in interpreters' workrelated well-being.

In general, though, research on affect within interpreting studies seems to inevitably bump into the "myth of neutrality", as discussed by Bontempo and Malcolm (2012, 109112; see also Diriker 2011). According to this myth, interpreters are professionals who do not influence the social interaction within which they interpret, nor should they be engaged in it, emotionally or otherwise (see Jacobsen 2013). Regardless of numerous alternative views of the interpreter's role, depicting interpreters as co-participants in the interaction they interpret (e.g. Wadensjö 1998; Angelelli 2004), the myth of the neutral, non-engaged, and emotionally detached interpreter may be one reason why there seems to be little research done on affect in the interpreting process. The research that has been conducted on the subject does, however, point to an important aspect of affect: it unfolds in and influences social interaction.

\section{Affect and the Experiencing and Narrating Self}

As discussed above, previous work conducted on affect in Translation Studies has emphasized either the physiological aspects of emotional reactions or the fact that affectivity makes the translator personally involved. Both of these lines of thinking, we argue, can be joined in our proposed concept of the translator's experiencing self, although in important respects it makes further contributions to a theory of affectivity as regards 
translating. In this section, we first define what we mean by affect, before we move on to describing the concepts of self and narrativity and the way these are related to our present argument.

Affect has been used to refer to a wide variety of meanings by different scholars, and related concepts such as emotion and feeling are given equally varied definitions (Wetherell 2012). In this paper, we employ Wetherell's definition of affect as being synonymous with “embodied meaning-making” $(2012,4)$. In other words, we see affect as the participants' emotional and bodily-experienced response to and interpretation of their lived experience, as a hinge between the self and the world. Thus, while affect involves the body and emotions, it does not stand in polar opposition to reason or cognition (see also Risku 2010). Both cognition and affect participate in processes of meaning-making, interpretation, and understanding. Where they differ, it has been suggested, is the extent to which they engage the body and the self:

[Emotions] are embodied thoughts, thoughts seeped with the apprehension that "I am involved." Thought/affect thus bespeaks the difference between a mere hearing of a child's cry and a hearing felt - as when one realizes that danger is involved or that the child is one's own.

(Rosaldo 1984, 143)

Affect as embodied meaning-making that engages the self should not, however, be understood as an inner process excluding the social surroundings of the individual, because the concept of the self is, itself, highly dependent on social contexts. Here, the self refers to an individual's own perception of him/herself (Mead 1937/1967). In other words, the self does not denote an unchanging, inner 'essence' of a person but our perception of ourselves, which is subject to change over time and across social contexts. The key question regarding the self, then, should not even be 'who we are' but 'when, where, and how we are' (Holstein and Gubrium 2000, 105, referring to Minh-ha 1992). Furthermore, the self is a social structure, as it develops only in social interaction (Mead 1937/1967). The attitudes that others have towards a person and the group attitudes prevalent in the social context surrounding him/her are essential for the development of the person's self (Mead 1937/1967, 158; Polkinghorne 1988, 149-150). Thus, “the general systematic pattern of 
social or group behavior [. . . ] enters as a whole into the individual's experience” (Mead 1937/1967, 158).

The day-to-day process of self-construction, in which individuals organize the group attitudes they come in contact with and formulate their perceptions of themselves, is rooted in narrative practice. We make sense of personally relevant experiences and events primarily by placing them into one storyline or another (Polkinghorne 1988, 150), and in "storying” our life experiences we simultaneously come to construct certain identities for ourselves (Holstein and Gubrium 2000, 107). Holstein and Gubrium (2000, 84) further argue that the spoken references to motives that individuals give to their actions, may, in time, become "actual components of the self.” People’s accounts of their actions are part of their self-construction. Importantly, the process of self-construction through narratives is simultaneously individually creative and socially constrained. Group attitudes and common storylines in the social context are used as interpretive resources for self-narratives. Thus, “[i]ndividuals compose their accounts” (Holstein and Gubrium 2000, 107, emphasis in the original). However, the composition always takes place "in the context of particular times and places; these circumstances influence how the self might be storied by presenting local relevancies” (Holstein and Gubrium 2000, 106).

Against this theoretical backdrop of the self and narrative, we underscore the importance of focusing on participants' narratives in the study of affects. As explained above, we understand affect as embodied meaning-making. This understanding focuses on conceptualizing affect with the help of both embodiment and meaning-making and it thus methodologically foregrounds narrative approaches. While the bodily reactions of affective experiences can also be studied independently of participants’ accounts, processes of meaning-making cannot be examined without attention to the subject who engages in that process, as the only realm of meaning that is directly accessible to us is our own (Polkinghorne 1988, 7), and narration provides avenues for investigating these realms. Furthermore, excluding narrativity from the study of affect and focusing exclusively on the physical realizations of affect would eclipse its social nature (Wetherell 2012, 75-76). Since this article aims at proposing avenues of research that would help bridge the gap between cognitive and sociological studies of translation, it seems meaningful to grasp precisely this interplay of internal emotions and the social context. 


\section{Examining Translating through Narratives of Affect: Three Cases}

In this section, we discuss three separate research projects or parts of research projects in order to trace some of the methodological implications and opportunities for translation research of treating affect as a lens through which translating is examined. As we will aim to show, translators' affective responses and accounts can be studied to gain insights into the way the activity of translating and participants' understanding of themselves as translators take place in a dialogue with cognitive processes and social contexts.

Tracing translators' feelings and affects may, in addition to observing the routines of the research participants, require that they are actively encouraged to verbalize their work and their emotional involvements. This can be done in several different ways. Below, we discuss three research projects that shed light on how affects can be studied. One project examines the role of laughter in building translators' shared professional identity (Koskinen 2008); the second investigates translators' technology acceptance through their imaginary love and break-up letters to various technological tools (Koskinen \& Ruokonen, in press); and the third explores the meaning and experience of volunteer church interpreting with autoethnography (Hokkanen, forthcoming).

These three cases were originally conducted separately, and with very different research questions in mind. We now revisit them from the point of view of how the emotional nature of these narratives can be used to uncover the dialogue of the cognitivepersonal and the social-interpersonal. In the following, we proceed from the most explicitly collective (group discussion) to the most individual (field journal), and from an analysis of a largely unconscious feature (laughter) to a method that requires extreme self-reflection from the part of the participant-researcher (autoethnography). In between these two extremes, we discuss a middle road: an analysis of emotions reported to the researcher (retrospective reflection) where social norms and expectations kick in.

\subsection{Laughter in EU Translators' Group Discussions}

Since affects are not only personal but also social and interactive, group contexts are a good source of affective data. In a study of Finnish translators in the European Commission (Koskinen 2008), focus groups were conducted to probe their identity and affiliations. In 
this data, laughter was such a dominant feature that it was deemed necessary to analyse it in more detail. As a physiological effect of affect, laughter is among those easily observable, but its functions and affective relations can be multifarious, and it thus requires a careful and contextually sensitive analysis. In interaction, laughter can be either shared or unilateral (i.e., one laughs but the others do not reciprocate), and shared laughter can be either volunteered or invited (speakers can invite laughter by starting to laugh themselves). The functions of laughter are similarly varied. In addition to simply being a response to jokes, funny stories, and witty remarks, laughter is a resource that can be strategically employed in interaction, for example in order to ease a delicate situation or hide embarrassment, to create a feeling of mutuality, or to single someone out (see Haakana 1999; Holmes 2000).

The focus of this article is on narrated affect. Laughter is an element of spoken narration, but in itself it is non-verbal, and it can be interpreted as a more direct effect of affect than reported verbalizations. Indeed, laughter can sometimes be an involuntary sign of affect, but it is necessary to keep in mind that laughter is also a strategic tool that people use to regulate interaction in social situations (Kangasharju \& Nikko 2009), and in that sense laughter has functions that are linked to the co-construction of particular narratives. For example, if the general tendency is towards shared views, shared laughter may encourage those with different views to also laugh along, instead of airing their disagreements. On the other hand, laughter can also indicate potential problem areas, since it is one way of displaying that the speaker wishes to cushion a delicate issue (Koskinen 2008, 114; Haakana 1999, 220). This interplay of laughter as a direct reaction to personally-felt affect on the one hand, and as a sign of affectivity as a social phenomenon on the other hand opens methodological opportunities, as the different kinds of laughter can be interpreted both as indications of cognitive states and as instances of strategic, albeit not necessary conscious, affective interaction, thus signalling the socially constructed understandings of the topics at hand.

In the study of EU translators, the participants were all drawn from an existing community. While it is necessary to acknowledge that the group sessions were not natural discussions, it seems reasonable to assume that maintaining face and keeping up relations with the colleagues was more relevant to the participants than 'posing' for the researcher. Although focus group sessions were not part of the everyday routine, discussions about 
work, at the workplace, with colleagues, became part of the lived experience of being 'at work'. Analysing elements of laughter in the context of the verbalized data and with reference to group dynamics can be used as cues to that experience.

In the study, shared and reciprocated laughter was found to be a dominant feature of the discussions. Although laughter was often invited by one of the participants, typically by laughing alone within or at the end of their turn, it was almost always reciprocated by others joining in and laughing along. In example 1 below (on the shared habit of reading a Finnish newspaper every day), there is little new information in any of the turns following H’s punch line, but each turn contributes to keeping the shared laughter alive.

\section{(Example 1)}

K: do you feel you have to do it, that it is sort of part of this, an obligation, that comes along this work or is it more related to your own free time and also to your Finnishness?

$\mathrm{H}: \quad$ well it is luckily not an obligation, if it was one would not get it done (shared laughter)

$\mathrm{K}: \quad$ one would not do it then (laughs)

L: $\quad$ one would not do (laughs)

I: if it was an obligation one would hardly do it first thing [in the morning H:

(shared laughter)

E: $\quad$ one would postpone it (--)

(shared laughter)

These jointly constructed instances, indicating a willingness to extend the humour, have been identified in other workplace settings as well, and laughing together has been found to be used to create and maintain solidarity and to contribute to social cohesion at work (Holmes 2000, 167). However, laughter has many roles. Findings among other professional groups such as social workers (Meyerson 1991) indicate that feelings of ambiguous identity or a sense of being caught in the middle can be relieved by resorting to humorous or cynical remarks. Similar ambiguities of selfhood and feelings of split loyalties are discernible in the context of EU translation. In many instances of laughter, there was a sarcastic and ironic undertone as the participants created emotional distance to the perceived ambiguities and discrepancies. Example 2 shows the usage of laughter to cushion a critical remark. 


\section{(Example 2)}

E: and then sometimes the experts in Finland give us totally peculiar (laughs), well suggestions that they definitely want us to use

Irony can help in creating space for dealing with contradictory demands by allowing some distance to the official policies and normative expectations. Seen in this light, cynicism is a positive force: it enables the speakers to recognize the contradictory nature of their work lives without having to attempt to resolve the contradictions; it allows "unresolvables, irreconcilables, and untenables to remain unresolved” (Meyerson 1991, 141). Irony and sarcasm seemed to have similar functions in these focus groups; it was not that the participants were attempting to laugh off some difficult issues. Instead, with the help of laughter they negotiated the amount of their personal involvement and maintained group cohesion in the face of potential disagreements (Koskinen 2008, 118).

Analysis of laughter attests to the affinities between the study of affects and methods such as ethnomethodology and conversation analysis (see Wetherell 2012, Ch. 4). A fine-tuned analysis of affective group interaction, as signalled here by the use of different types of laughter, can be used to identify the issues that are expected to be shared and to pinpoint areas of discontent and ambiguity in translators' or interpreters' work-related contexts. As a method, the analysis of laughter does not require any degree of reflexivity from the participants, quite the opposite: the less they consciously monitor their use of laughter, the more revealing the analysis of the affective stances can become.

\subsection{Translators' Love and Break-up Letters}

The structuring role of laughter in the group sessions discussed above was unanticipated in the research design, and its explanatory power in understanding the affective bonds of the participants only became evident during data analysis. In our second example, the love letter/break-up letter method, the intention to tap into the affective commitments of the participants is explicit, as they are invited to narrate their strongest personal emotions in a personifying letter format. 
The method was originally designed for usability research to investigate user experiences. The second author of this paper used it to uncover translators' emotional involvement with their work, particularly their tools, to better understand issues of technology acceptance in a rapidly changing field (Koskinen and Ruokonen, in press). The design was three-dimensional in involving an international group of EU translators $(n=44)$, practicing Finnish translators $(\mathrm{n}=26)$ and advanced translation students in Finland and Ireland ( $\mathrm{n}=33)$. They wrote a total of 148 letters.

The task is simple: the participants are asked to write a love letter or break-up letter to a designated object. The object can be predetermined, but in this case the respondents were free to choose the tool or aspect of their work to which they wanted to write their letters. This freedom allowed us to analyse not only how translators report feeling about particular translation tools but also how and whether these tools figure in the bigger picture. In other words, the personified addressees of these letters were interpreted as having been selected because of being central to the respondents' positive or negative experiences in their everyday work, and the expression of a negative or positive affect, or both, was thus seen as an indicator of relevance.

Letters were written individually, and no discussion or disclosure to colleagues was involved. This method is thus less explicitly social than the group discussions introduced above. However, a social bond still exists between the respondent and the researcher, and the fact that the letters, although ostensibly written to the object of emotion, are in effect written to the researcher is likely to inform the selection of topics and the writing process. Some letters even include (affective) guidelines for their interpretation directed to the researcher (see Example 3). This social bond needs to be taken into account in the analysis, but it can also be taken up analytically to investigate what kinds of emotions and which narratives of translation work are socially acceptable.

(Example 3)

I hate you, D.O.D, $* *$

Because you prevent me from doing my best, you stop my creativity as your terminology is out-of-date and I have to convey it through new texts... Go away, be burned, fly to the space! 


\section{** DIRTY OLD DIRECTIVES}

Please consider these answers in their context: a rainy Monday, a long amended directive to translate and some (frustrating) years behind... (EU-31)

Compared to the analysis of laughter, this method builds on a degree of reflexivity. Writing a fictional letter is clearly set apart from regular work day activities, and it invites a conscious analysis of past events and the respondents' affective involvement in them, as narrated to an outside observer. Letters as a genre offer an available repertoire for the respondents, and as professional text workers translators are naturally well able to toy with it. The clearly playful research design further supports this attitude. The letter format and the binary structure of the set-up also encourage exaggeration of emotion. Some caution is thus needed in drawing conclusions. This method cannot be considered a window to actually felt emotions, but a tool for eliciting dramatized stories of affective involvements.

The analysis of the letter data is still in progress. Among the findings so far is that translators' attitudes towards technology are far less negative than is sometimes depicted. They appreciate their efficient tools and have little nostalgic desire to go back to former times. However, as their technologized work environment is vulnerable to malfunctioning technology, the resulting lack of efficiency produces strong negative affects and most likely leads to reduced job satisfaction. The fictional letters are the participants' accounts of past events and past emotional stances. Narratives of this kind, Wetherell $(2012,89)$ argues, "are likely to become more important as the body winds down and as the moment of strong affect is carried forward as a memory or story, with new accompanying affect.” The method functions as a magnifying glass for the lived experience, pushing it to one of the two extremes and creating new affective layers of remembering, reflecting, and reporting.

\subsection{Autoethnography of Church Interpreting}

The third and final case of viewing translating through the lens of affect comes from an autoethnographic study on simultaneous church interpreting in Finnish Pentecostal churches (Hokkanen, forthcoming). In this methodology, the first author of this paper used her social position as a church member and volunteer interpreter to elicit data on the meaning and experience of the church’s simultaneous interpreting practice. However, also 
being a professionally trained translator and interpreter, her identities as a volunteer interpreter, professional translator/interpreter, and interpreting researcher all came into play in the research process (cf. Bahadır 2004), often creating tensions that upon further analysis revealed aspects of each social world that she had not been as keenly aware of before. Thus, autoethnography allowed the author to interrogate emotions and embodied experiences she had 'in the field' in order to arrive at a deeper understanding of the social world of the church (the main object of research) and that of professional interpreting and interpreting research.

In autoethnography, the researcher's emotional experiences in the field are turned into entries in the field journal, which subjects his/her affects to a process of verbalization and narration. (See Ellis and Bochner 2000 for a further introduction to autoethnography.) In practice, following the ethnographic tradition, the first author gathered fieldnotes on the occasions when she interpreted at church or when her identity as a church interpreter came to bear on a social situation. These preliminary fieldnotes, especially when gathered by hand in the interpreting booth in the middle of interpreting a church service, were very succinct "scratch notes" (Ottenberg 1990, 148; see Example 4). These often hastily-written and cryptic notes were subsequently elaborated into a fuller narrative as an entry in the field journal (see Example 5). ${ }^{3}$

(Example 4)

[Name of Speaker 1] (?) :) intonation

[Name of Speaker 2] crying

\section{(Example 5)}

Next the host of the service introduces a man who gives a testimony, a person who is well-known and influential in his home town. I'm not sure I catch his name right. Says that people who knew him were very surprised that [Speaker 1] had come to faith. He is a lively and articulate speaker, and I feel I have to put some extra effort into matching his vivacious intonation, even though I believe to be far from monotonous when I interpret. [Speaker $1]$ is an extremely captivating speaker and I smile when he finishes.

\footnotetext{
${ }^{3}$ The fieldnotes were originally written in Finnish with occasional English code-switching, marked in the examples by the use of italics. The examples were translated into English by the first author.
} 
Then the host introduces another person giving a testimony. [Speaker 2]'s cancer was healed. (I couldn't find the equivalent for metastasis, so I went around it saying the cancer "had spread into three places" on her body.) It couldn't be operated, but God healed her. The host interviews [Speaker 2], and soon she starts to cry while giving her testimony. I try to sound empathetic, but don't cry, myself. After the testimony, I don't exactly feel fake, but I realize I relayed emotion without it going very deep in my own heart - this time.

As a process of narrative construction based on affective experiences, the writing of the fieldnotes was naturally conditioned by the socially available resources with which the first author could express and understand those experiences. For example, she would describe in the fieldnotes affective experiences of restfulness and relaxation taking place in the interpreting booth, using socially available concepts to interpret those emotions. Thus, instead of simply writing about feeling at ease, she would write about feeling the presence of the Holy Spirit.

Affect was not an explicit focus of the study to begin with, but its importance for both fieldwork and for the examination of social meanings related to church interpreting became increasingly pronounced as the study progressed. Ethnographic fieldwork as a method requires the physical presence of the researcher in a social context, but, in fact, it is dependent on embodiment even beyond mere presence; “[p]hysical and sensuous presence [...] allows observation and witness and the use of five-sense channels for recording data relating to social atmosphere, emotional colour and unspoken assumptions” (Willis 2000, xiii). Thus, ethnographers not only observe participants' emotional reactions, but may analyse their own affective experiences in a process of building a deeper understanding of the social context. Moreover, as autoethnography emphasizes the role of the researcher's personal experience as part of the research data (Ellis and Bochner 2000), the author's personal affective experiences related to church interpreting were incorporated into the study.

Because the affective narratives recorded in the fieldnotes were experienced firsthand by the first author, their analysis differed from methodologies involving affective narratives written by others. Revisiting accounts of personal affective experiences often made the author also relive the related emotions. The body remembered and reacted, 
whether the fieldnotes described embarrassment over interpreting errors, discomfort over conflicting identities, or tearfulness over feeling loved and accepted in the midst of a religious experience. As part of the analysis of such affective accounts, some of the fieldnotes were rewritten into short narratives in the style of creative non-fiction and poetry, following the example set by autoethnographers who mix different writing genres in reporting their studies. Thus, narratives were used not only as a means of reporting affective experiences but also as a means of analysis, in an effort to understand those experiences in light of the social context (cf. Spry 2001).

In this autoethnographic study, the analysis of narratives describing personal affective experiences demanded a high degree of reflexivity, but it revealed how certain values and norms from different social worlds moulded the first author's practice and experience of interpreting at church. Having been socialized into professional interpreting through a university education and into the religious setting through active membership, the author had internalized certain values and norms from these social worlds, some to the extent that she had become unaware of them guiding her interpreting. Analysing the affective reactions occurring while simultaneously interpreting church services assisted the author in making her (again) aware of these values and norms and of the way they contributed to her interpreting practice. By beginning from affects, the author was able to analyse issues such as the importance of preparation for interpreting practice and for religious experience, or the opposite pulls of a neutral interpreter role and a fully-involved church member engaging in the interpreted service (Hokkanen 2013).

\section{Conclusion}

A focus on translators' affects, understood as their embodied meaning-making and operationalized with narratives, offers a promising avenue for understanding and analysing their experiencing self as an interface where the cognitive and the social are brought together and made sense of through the translator's affective engagement with them. Importantly, we do not wish to propose an interpretation of affect as being opposed to or separate from reason or cognition but as integrally linked to it. We see affect as meaningmaking that involves the self of the individual: we experience affects when we interpret that certain aspects or events observed in the social world have become personally relevant. 
The three cases discussed in this paper point to the ways in which narrated affect allows us to interpret how translators engage with both social and cognitive aspects of their work. The cases were selected to illustrate some of the opportunities provided by incorporating affect into different methodological frameworks and research designs. By subjecting laughter to scrutiny as a non-verbal display of affect in a group context (section 4.1), we were able to trace the ways in which it signals socially constructed understandings of work-related topics (such as professional identity) and allows the researcher to identify moments of tension between the internal and external pressures.

In our second case (section 4.2), the use of dramatized emotional letters that translators were asked to write, addressed to any tool or aspect of their work, was founded upon the assumption that translators do, in fact, engage with their work emotionally. Indeed, while not providing a direct window into actual, spontaneous affective experiences, the letters show how the respondents placed their past experiences into an affective storyline, emphasizing either affection or dislike towards the chosen addressee. This kind of forced affective assessment may best function as a component of sociological studies, opening avenues from workplace observations towards lived experiences, and also potentially challenging any preconceived understandings of translators' affects (in this case, for example, the notion of translators being technology-averse). It might also shed new light to cognitive, process-oriented studies. For example, the measurement of task times tends to be combined with assumptions of efficiency or difficulty, whereas affective narratives of the task might bring in other explanatory aspects such as pleasure.

In our final case (section 4.3), we used narrated affective experiences in the form of fieldnotes in an analysis of social norms and values related to interpreting. With this autoethnographic research design, we were able to illustrate how the interpreter's affects were intertwined with the social surroundings and with the cognitive processes related to interpreting, such as monitoring the use of voice or making concrete translational decisions. Nevertheless, the focus of autoethnography has traditionally been on the study of social meanings and processes of identity construction (see e.g. Bochner and Ellis 2002), rather than on cognitive functions. Thus, also in the case discussed here, the autoethnographer's affective narratives shed more light on the social than the cognitive aspects of interpreting. 
With our title we suggest that affect can be seen as a hinge between the experiencing self and its surrounding social context. As can be seen from the discussion of the three cases above, we both tend to lean towards explaining the social with the help of narrated affect due to our personal research interests. It is important to emphasize, however, that the 'hinge' is not an isolated component that would separate two distinct entities - the social and the cognitive. Rather, we suggest that affect is intricately intertwined with both social and cognitive processes, which, in turn, do not stand in a dichotomous relationship but are highly interconnected. Indeed, it is precisely this embeddedness of affect within both the social and the cognitive, we argue, that makes it a methodologically useful starting point in studies wishing to extend their analysis to both experiencing selves and the surrounding social contexts.

Our approach to affect in this paper has been based on narratives, but, as we have pointed out, it is not the only approach, nor is it without its limitations. In our daily life, the amount of our conscious reflection shifts as "we move in and out of 'knowing' what we are about” (Wetherell 2012, 129). Narratively attuned research methods such as those described above can be used to promote reflection, but it is important to notice that once we move into conscious narrating of affect, these accounts are not retrievals of personal affect but in fact new forms of social action (Wetherell 2012, 129). While one needs to have an understanding of the nature of narrative accounts and thus also the limits of methods focusing on participants' accounts, we argue, with Wetherell (2012, 20), that "human affect is inextricably linked with meaning-making and with the semiotic (broadly defined) and the discursive” and that this meaning-making is, in fact, what research will need to aim to understand.

In this article, we had a very pragmatic aim. We wanted to emphasize the role of affect in understanding how translators experience the situations they are in, and we wanted to explore the possibilities of studying this empirically. The three cases described above are only partial and preliminary answers to the "huge theoretical and practical challenge" (Wetherell 2012, 11) that affect presents to researchers. We hope that sharing our personal experiences will inspire other scholars to experiment with these and other methods and so increase our understanding of translators' embodied meaning-making of translating in different social contexts. 


\section{References}

Angelelli, Claudia. 2004. Medical Interpreting and Cross-Cultural Communication. London: Cambridge University Press.

Bahadır, Şebnem. 2004. "Moving in-between: The Interpreter as Ethnographer and the Interpreting-Researcher as Anthropologist.” Meta 49 (4): 805-821.

Baraldi, Claudio, and Laura Gavioli. 2007. “Dialogue Interpreting as Intercultural Mediation: An Analysis in Healthcare Multicultural Settings. In Dialogue and Culture, edited by Marion Grein, and Edda Weigand, 155-176. Amsterdam \& Philadelphia: John Benjamins.

Bochner, Arthur P., and Carolyn Ellis (eds). 2002. Ethnographically Speaking: Autoethnography, Literature, and Aesthetics. Walnut Creek: AltaMira.

Bontempo, Karen, and Karen Malcolm. 2012. “An Ounce of Prevention Is Worth a Pound of Cure: Educating Interpreters about the Risk of Vicarious Trauma in Healthcare Settings.” In In Our Hands: Educating Healthcare Interpreters, edited by Laurie Swabey, and Karen Malcolm, 105-130. Washington, D.C.: Gallaudet University Press.

Cirillo, Letizia. 2010. “Managing Affect in Interpreter-Mediated Institutional Talk: Examples from the Medical Setting.” Journal of Specialised Translation 14: 55-79.

Diriker, Ebru. 2011. “Agency in Conference Interpreting: Still a Myth?” Gramma: Journal of Theory and Criticism 19: 27-36.

Ellis, Carolyn, and Arthur P. Bochner. 2000. “Autoethnography, Personal Narrative, Reflexivity: Researcher as Subject.” In Handbook of Qualitative Research, Second edition, edited by Norman K. Denzin, and Yvonna S. Lincoln, 733-768. Thousand Oaks, London \& New Delhi: Sage.

Haakana, Markku. 1999. Laughing Matters. A Conversation Analytical Study of Laughter in Doctor-Patient Interaction. Doctoral dissertation, University of Helsinki.

Hokkanen, Sari. 2013. “Tulkki keskellä hengellistä kokemusta: Simultaanitulkkaus Tampereen helluntaiseurakunnan kokouksessa [The interpreter amidst religious experience: Simultaneous interpreting in the Tampere Pentecostal Church]”. In 
Tulkattu Tampere, edited by Kaisa Koskinen, 263-284. Tampere: Tampere University Press.

Hokkanen, Sari. (forthcoming) To Serve and to Experience: An Autoethnographic Study of Simultaneous Church Interpreting. Doctoral dissertation, University of Tampere.

Holmes, Janet. 2000. "Politeness, Power and Provocation: How Humour Functions in the Workplace.” Discourse Studies 2 (2): 159-185.

Holstein, James A., and Jaber F. Gubrium. 2000. The Self We Live By: Narrative Identity in a Postmodern World. New York \& Oxford: Oxford University Press.

Hubscher-Davidson, Séverine. 2011. “A Discussion of Ethnographic Research Methods and Their Relevance for the Translation Process.” Across Languages and Cultures 12 (1): $1-18$.

Hubscher-Davidson, Séverine. 2013a. “The Role of Intuition in the Translation Process: A Case Study.” Translation and Interpreting Studies 8 (2): 211-232.

Hubscher-Davidson, Séverine. 2013b. "Emotional Intelligence and Translation Studies: A New Bridge.” Meta 58 (2): 324-346.

Jacobsen, Bente. 2013. "Training the Trainers: Dealing with Interpreting Ethics.” In Training the Trainers: Nordic Seminar on Interpreter Education, edited by Cecilia Wadensjö, 38-47. Stockholm: Tolk- och översättarinstitutet, Stockholm University. Kangasharju, Helena, and Tuija Nikko. 2009. "Emotions in Organizations: Joint Laughter in Workplace Meetings.” International Journal of Business Communication 46 (1): $100-119$.

Koskinen, Kaisa. 2008. Translating Institutions: An Ethnographic Study of EU Translation. Manchester: St. Jerome.

Koskinen, Kaisa. 2012. "Domestication, Foreignization and the Modulation of Affect.” In Domestication and Foreignization in Translation Studies, edited by Hannu Kemppanen, Marja Jänis, Alexandra Belikova, 13-32. Berlin: Frank \& Timme. Koskinen, Kaisa, and Minna Ruokonen. (in press) “Love Letters or Hate Mail? Translators' Technology Acceptance in the Light of their Emotional Narratives.” In Human Issues in Translation Technology, IATIS Yearbook 2015, edited by Dorothy Kenny. London \& New York: Routledge. 
Lehr, Caroline. 2014. “The Relevance of Emotion for Professional Translation and Translation Studies.” In Man vs. Machine? The Future of Translators, Interpreters and Terminologists: Proceedings of the XXth FIT World Congress, Berlin 2014, edited by Wolfram Baur, Brigitte Eichner, Sylvia Kalina, Norma Keßler, Felix Mayer, and Jeannette Ørsted, 601-606. Berlin: BDÜ Fachverlag.

Mead, George H. 1937/1967. Mind, Self, and Society: From the Standpoint of a Social Behaviorist. Chicago: University of Chicago Press.

Meyerson, Debra E. 1991. “’Normal Ambiguity?’ A Glimpse of an Occupational Culture.” In Reframing Organizational Culture, edited by Peter J. Frost, Larry F. Moore, Meryl Reis Luis, Craig C. Lundberg, and Joanne Martin, 131-156. Newbury Park: Sage.

Minh-ha, Trinh. 1992. Framer Framed. New York: Routledge.

O’Brien, Sharon. 2011. “Introduction.” In Cognitive Explorations in Translation: Eyes, Keys, TAPs. IATIS Yearbook 2010, edited by Sharon O’Brien, 1-14. London \& New York: Continuum.

Ottenberg, Simon. 1990. “Thirty Years of Fieldnotes: Changing Relationships to the Text.” In Fieldnotes: The Makings of Anthropology, edited by Roger Sanjek, 139-160. Ithaca, NY \& London: Cornell University.

Pérez-González, Luis. 2012. “Amateur Subtitling and the Pragmatics of Spectatorial Subjectivity.” Language and Intercultural Communication 12 (4): 335-352.

Polkinghorne, Donald. 1988. Narrative Knowing and the Human Sciences. Albany, NY: State University of New York Press.

Risku, Hanna. 2002. “Situatedness in Translation Studies.” Cognitive Systems Research 3: 523-533.

Risku, Hanna. 2010. “A Cognitive Scientific View on Technical Communication and Translation: Do Embodiment and Situatedness Really Make a Difference?” Target 22 (1): 94-111.

Robinson, Douglas. 1991. The Translator's Turn. Baltimore \& London: The Johns Hopkins University Press.

Rosaldo, Michelle. Z. 1984. “Toward an Anthropology of Self and Feeling.” In Culture Theory: Essays on Mind, Self, and Emotion, edited by Richard A. Shweder, and 
Robert A. LeVine, 135-157. Cambridge, London, New York, New Rochelle, Melbourne \& Sydney: Cambridge University Press.

Rossini, Linda, and Delia Chiaro. 2010. “Audiences and Translated Humour: An Empirical Study.” In Translation, Humour and the Media Vol 2, edited by Delia Chiaro, 121-137. London \& New York: Continuum.

Spry, Tami. 2001. “Performing Autoethnography: An Embodied Methodological Praxis.” Qualitative Inquiry 7: 706-732.

Tirkkonen-Condit, Sonja, and Johanna Laukkanen. 1996. "Evaluations - A Key towards Understanding the Affective Dimension of Translational Decisions.” Meta 41 (1): 45-59.

Wadensjö, Cecilia. 1998. Interpreting as Interaction. London \& New York: Longman. Wetherell, Margaret. 2012. Affect and Emotion: A New Social Science Understanding. London, Thousand Oaks \& New Delhi: Sage.

Willis, Paul. 2000. The Ethnographic Imagination. Cambridge \& Malden: Polity. 